\title{
Small Area Disease Risk Estimation and Visualization Using $R$
}

by Paula Moraga

\begin{abstract}
Small area disease risk estimation is essential for disease prevention and control. In this paper, we demonstrate how $\mathrm{R}$ can be used to obtain disease risk estimates and quantify risk factors using areal data. We explain how to define disease risk models and how to perform Bayesian inference using the INLA package. We also show how to make interactive maps of estimates using the leaflet package to better understand the disease spatial patterns and communicate the results. We show an example of lung cancer risk in Pennsylvania, United States, in year 2002, and demonstrate that R represents an excellent tool for disease surveillance by enabling reproducible health data analysis.
\end{abstract}

\section{Introduction}

Disease risk mapping analyses can help to better understand the spatial variation of the disease, and allow the identification of important public health determinants. These analyses are essential to inform programmes of disease prevention and control. The increased availability of geospatial disease and population data has enabled to study a number of health outcomes worldwide such as influenza and cancer in developed countries (Moraga and Ozonoff, 2013; Moraga and Kulldorff, 2016), and neglected tropical diseases (Moraga et al., 2015; Hagan et al., 2016).

Areal disease data often arise when disease outcomes observed at point level locations are aggregated over subareas of the study region due to several reasons such as patient confidentiality. Producing disease risk estimates at areal level is complicated by the fact that raw rates can be very unstable in areas with small populations and for rare diseases, and also by the presence of spatial correlation that may exist due to spatially correlated risk factors (Leroux et al., 2000). Thus, generalized linear mixed models are often used to obtain disease risk estimates since they enable to improve local estimates by accommodating spatial correlation and the effects of explanatory variables. Bayesian inference in these models may be performed using the Integrated Nested Laplace Approximation (INLA) approach (Rue et al., 2009) which is a computational alternative to MCMC that allows to do approximate Bayesian inference in latent Gaussian models. This approach is implemented in the $\mathrm{R}$ package called INLA (Rue et al., 2017) (http: //www. r-inla.org/).

Small area disease estimates can be visualized through maps, greatly facilitating effective communication. $R$ provides excellent tools for visualization including packages for making interactive maps such as leaflet (Cheng et al., 2017). The maps created with leaflet support interactive panning and zooming which is very convenient to examine small areas in detail.

In this paper, we illustrate the use of $\mathrm{R}$ for performing disease risk mapping analysis using areal data. First, we introduce disease risk models for areal data and give a brief overview of INLA. In Section 2.4 we show how to estimate lung cancer risk and quantify risk factors in Pennsylvania, United States, in year 2000. Specifically, we discuss how to compute the observed and expected disease counts in the Pennsylvania counties, how to obtain disease risk estimates by fitting a spatial disease risk model using INLA, and how to build interactive maps showing the risk estimates using leaflet. Finally, the conclusions are presented.

\section{Disease risk models}

Disease risk estimates in areas can be obtained by computing the Standardized Incidence Ratios (SIRs). For area $i, i=1, \ldots, n$, the SIR is obtained as the ratio of the observed to the expected disease counts: $\operatorname{SIR}_{i}=Y_{i} / E_{i}$. The expected counts represent the total number of disease cases that one would expect if the population of the specific area behaved the way the standard (or regional) population behaves. The expected counts can be calculated using indirect standardization as

$$
E_{i}=\sum_{j=1}^{m} r_{j}^{(s)} n_{j},
$$

where $r_{j}^{(s)}$ is the disease rate in stratum $j$ of the standard population, and $n_{j}$ is the population in stratum $j$ of the specific area. The SIR corresponding to area $i, \operatorname{SIR}_{i}$, indicates whether the area $i$ has more $\left(\mathrm{SIR}_{i}>1\right)$, equal $\left(\mathrm{SIR}_{i}=1\right)$ or fewer $\left(\mathrm{SIR}_{i}<1\right)$ cases observed than expected from the standard population. When applied to mortality data, the ratio is commonly known as the Standardized 
Mortality Ratio or SMR.

Although in some situations SIRs can give a sense of the disease's spatial variability, very extreme values can occur in areas with small populations owing to the small sample sizes involved. In contrast, disease models are preferred to obtain disease risks estimates because they enable to incorporate covariates and borrow information from neighboring areas to improve local estimates, resulting in the smoothing or shrinking of extreme values based on small sample sizes (Gelfand et al., 2000). A common approach is to model the observed counts $Y_{i}, i=1, \ldots, n$, using a Poisson distribution with mean $E_{i} \times \theta_{i}$, where $E_{i}$ is the expected counts and $\theta_{i}$ is the relative risk in area $i$. Then, the log risks are modeled with a sum of an intercept to model the overall disease risk level, and random effects that account for extra-Poisson variability in the observed data (Lawson, 2009). Areas with relative risks $\theta>1$ and $\theta<1$ are areas with high and low risks, respectively. Areas with $\theta=1$ have the same risk as expected from the standard population.

The general model in disease mapping is expressed as

$$
\begin{gathered}
Y_{i} \sim \operatorname{Po}\left(E_{i} \times \theta_{i}\right), i=1, \ldots, n, \\
\log \left(\theta_{i}\right)=\alpha+u_{i}+v_{i} .
\end{gathered}
$$

Here, $\alpha$ denotes the overall risk level, $u_{i}$ is a spatial structured random effect that models the spatial dependence between the relative risks, and $v_{i}$ is an unstructured exchangeable random effect that models uncorrelated noise. Often, other covariates or random effects are also included to quantify risk factors and deal with other sources of variability.

A model commonly used in disease mapping is the Besag-York-Mollié (BYM) model (Besag et al., 1991). In this model, the spatially structured component $u_{i}$ is modelled with the conditional autoregressive (CAR) distribution which smoothes the data according to a certain adjacency structure given by a neighborhood matrix that specifies two areas are neighbours if they have a common boundary. The CAR distribution is expressed as

$$
u_{i} \mid \mathbf{u}_{-\mathbf{i}} \sim N\left(\bar{u}_{\delta_{i}}, \frac{\sigma_{u}^{2}}{n_{\delta_{i}}}\right),
$$

where $\bar{u}_{\delta_{i}}=n_{\delta_{i}}^{-1} \sum_{j \in \delta_{i}} u_{j}$, and $\delta_{i}$ and $n_{\delta_{i}}$ represent, respectively, the set of neighbours and the number of neighbours of area $i$. The unstructured component $v_{i}$ is modelled using independent and identically distributed normal variables with zero-mean and variance equal to $\sigma_{v}^{2}$.

\section{INLA}

Traditionally, Bayesian inference has been implemented via MCMC methods which make inference tractable for complex models but may present convergence and computation time problems. Integrated Nested Laplace Approximation (INLA) is a computational less-intensive alternative to MCMC designed to perform approximate Bayesian inference in latent Gaussian models (Rue et al., 2009). These models include a very wide and flexible class of models ranging from generalized linear mixed to spatial and spatio-temporal models. Specifically, models are of the form

$$
\begin{aligned}
& y_{i} \mid \mathbf{x}, \boldsymbol{\theta} \sim \pi\left(y_{i} \mid x_{i}, \boldsymbol{\theta}\right), \\
& \mathbf{x} \mid \boldsymbol{\theta} \sim N\left(\mathbf{0}, \boldsymbol{Q}(\boldsymbol{\theta})^{-1}\right), \\
& \boldsymbol{\theta} \sim \pi(\boldsymbol{\theta}),
\end{aligned}
$$

where $\mathbf{y}$ are the observed data, $\mathbf{x}$ represents a Gaussian field, and $\boldsymbol{\theta}$ are hyperparameters. Observations $y_{i}$ are assumed to belong to an exponential family with mean $\mu_{i}=g^{-1}\left(\eta_{i}\right)$. The linear predictor $\eta_{i}$ accounts for effects of various covariates in an additive way,

$$
\eta_{i}=\alpha+\sum_{k=1}^{n_{\beta}} \beta_{k} z_{k i}+\sum_{j=1}^{n_{f}} f^{(j)}\left(u_{j i}\right) .
$$

Here, $\alpha$ is the intercept, $\left\{\beta_{k}\right\}$ 's quantify the linear effects of covariates $\left\{z_{k i}\right\}$ on the response, and $\left\{f^{(j)}(\cdot)\right\}^{\prime}$ 's are a set of non-linear or smooth functions defined in terms of some covariates $\left\{u_{j i}\right\}$. This formulation permits to accommodate a wide range of models thanks to the very different forms that the functions $\left\{f^{(j)}\right\}$ can take including the disease risk models previously introduced. INLA uses a combination of analytical approximation and numerical integration to obtain approximated posterior distributions of the parameters that can then be post-processed to compute quantities of interest like posterior expectations and quantiles. 
The INLA approach is implemented in the R package INLA. This package is not on CRAN because it uses some external $\mathrm{C}$ libraries that make difficult to build the binaries. Therefore, when we install the package we need to use install . packages() adding the URL of the INLA repository:

install. packages ("INLA", repos = "https://inla.r-inla-download.org/R/stable", dep $=$ TRUE)

To fit a model using INLA we need to take two steps. First, we need to write the linear predictor of the model as a formula object in R. Then, we run the model calling the inla() function where we specify the formula, the family, the data and other options. Results can be inspected with the summary() function and the posterior distributions can be post-processed using a set of specific functions provided by INLA. Further details about how to use all these functions will be given in the disease mapping example in next Section.

\section{Example: lung cancer risk in Pennsylvania}

In this Section we present an example of small area disease mapping study where we estimate the risk of lung cancer in Pennsylvania counties in year 2002. We use the data contained in the R package SpatialEpi (Kim and Wakefield, 2016). The data contain the counties population which was obtained from the 2000 decennial census, and the lung cancer and smoking proportions which were obtained from the Pennsylvania Department of Health. We show how to calculate the observed and expected disease cases, and the the SIRs in each of the counties. We also obtain disease risk estimates and quantify risk factors by fitting a Bayesian model using INLA. Finally, we show how to make interactive maps of the risk estimates using leaflet.

\section{Data}

We start by loading the SpatialEpi package and attaching the pennLC data.

library(SpatialEpi)

data(pennLC)

By typing ?pennLC we see pennLC is a list with the following elements:

- geo: a data frame of county ids, and longitude and latitude of the geographic centroid of each county,

- data: a data frame of county ids, number of cases, population and strata information,

- smoking: a data frame of county ids and proportion of smokers,

- spatial.polygon: a SpatialPolygons object with the map of Pennsylvania.

pennLC $\$$ data contains the number of lung cancer cases and the population at county level, stratified on race (white and non-white), gender (female and male) and age group (under 40, 40-59, 60-69 and 70+).

We now create a data frame called $d$ with columns containing the counties ids, the observed and expected number of cases, the smoking proportions and the SIRs. Specifically, $d$ will contain the following columns:

- id: id of each county,

- Y: observed number of cases in each county,

- E: expected number of cases in each county,

- smoking: smoking proportion in each county,

- SIR: SIR of each county.

\section{Observed cases}

pennLC $\$$ data contains the cases in each county stratified by race, gender and age. We can obtain the number of cases in each county, $Y$, by aggregating the rows of pennLC $\$$ data by county and adding up the observed number of cases.

$$
\begin{gathered}
\mathrm{d}<-\operatorname{aggregate}(\mathrm{x}=\text { pennLC} \$ \text { data } \$ \text { cases }, \text { by }=1 \text { ist }(\text { county }=\text { pennLC } \$ \text { datatacounty }), \\
\text { FUN }=\text { sum })
\end{gathered}
$$

aggregate() returns a data frame where the first row is the county and the second column is the observed number of cases in each of the counties. We set the column names of the returned object equal to id and $Y$.

names $(d)<-c(" i d ", " Y ")$ 


\section{Expected cases}

Now we calculate the indirectly standardized expected number of cases in each county as explained in Section 2.2. That is, we use the strata-specific rates from the the Pennsylvania population (standard population), and apply them to the population distribution of the county. The expected counts represent the total number of disease cases one would expect if the population in the county behaved the way the Pennsylvania population behaves. We can do this by using the expected() function of SpatialEpi. This function has three arguments, namely,

- population: a vector of population counts for each strata in each area,

- cases: a vector with the number of cases for each strata in each area,

- n.strata: number of strata considered.

Vectors population and cases have to be sorted by area first and then, within each area, the counts for all strata need to be listed in the same order. All strata need to be included in the vectors, including strata with 0 cases. Hence, to get the expected counts we first sort the data using the order () function where we specify the order as county, race, gender and finally age.

pennLC $\$$ data <- pennLC $\$$ data[order (pennLC $\$$ data $\$$ county, pennLC $\$$ datatarace, pennLC\$data\$gender, pennLC\$data\$age), ]

Then we call the expected() function to obtain the expected counts $E$ in each county. In the function we set population equal to pennLC $\$$ data $\$$ population and cases equal to pennLC $\$$ data $\$$ cases. There are 2 races, 2 genders and 4 age groups for each county, so number of strata is set to $2 \times 2 \times 4=16$.

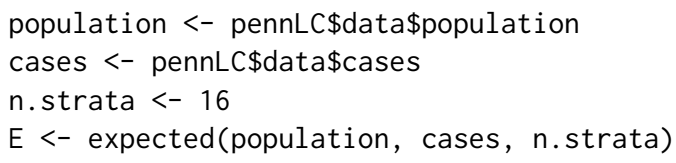

Now we add the vector $E$ to the data frame $d$ which contains the counties ids (id) and the observed counts $(Y)$, making sure the E elements correspond to the counties in $d \$ i d$ in the same order. To do that, we use match() to calculate the vector of the positions that match $d \$ i d$ in unique (pennLC $\$$ data $\$$ county) which are the corresponding counties of $E$. Then we rearrange $E$ using that vector.

$d \$ E<-E[m a t c h(d \$ i d$, unique $($ pennLC $\$$ datatacounty $))]$

\section{Smokers proportions}

We also add to $d$ the variable smoking which represents the proportion of smokers in each county. We add this variable using the merge() function where we specify the columns for merging as id in $d$ and county in pennLC\$smoking.

$d<-\operatorname{merge}(d$, pennLC\$smoking, by. $x=$ "id", by. $y=$ "county")

\section{SIRs}

Finally, we compute the vector of SIRs as the ratio of the observed to the expected counts, and add it to the data frame $d$.

$\mathrm{d} \$ S I R<-d \$ Y / d \$ E$

\section{Add data to map}

The map of Pennsylvania counties is given by the SpatialPolygons object called pennLC \$spatial . polygon. Using this object and the data frame $d$ we can create a SpatialPolygonsDataFrame called map, that will allow us to make maps of the variables in d. In order to do that, we first set the row names of the data frame $d$ equal to $d \$ i d$. Then we merge pennLC\$spatial . polygon and d matching the SpatialPolygons member Polygons ID slot values with the data frame row names.

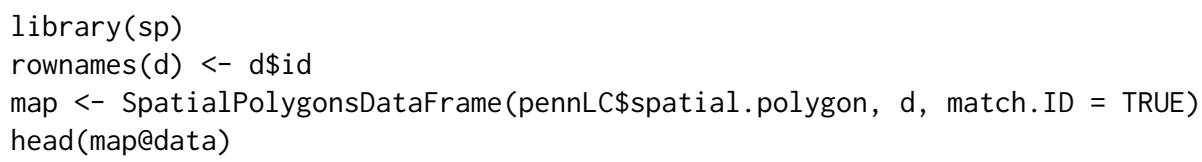




$\begin{array}{lrrrrrr}\text { \#\# } & \text { id } & \text { Y } & \text { E smoking } & \text { SIR } \\ \text { \#\# adams } & \text { adams } & 55 & 69.62730 & 0.234 & 0.7899200 \\ \text { \#\# allegheny } & \text { allegheny } & 1275 & 1182.42804 & 0.245 & 1.0782897 \\ \text { \#\# armstrong } & \text { armstrong } & 49 & 67.61012 & 0.250 & 0.7247435 \\ \text { \# beaver } & \text { beaver } & 172 & 172.55806 & 0.276 & 0.9967660 \\ \text { \#\# bedford } & \text { bedford } & 37 & 44.19013 & 0.228 & 0.8372910 \\ \text { \#\# berks } & \text { berks } & 308 & 300.70598 & 0.249 & 1.0242563\end{array}$

\section{Mapping variables}

We can visualize the observed and expected disease counts, the SIRs, as well as the smokers proportions in an interactive chropleth map using the leaflet package. We create the map by first calling leaflet() and adding the default OpenStreetMap map tiles to the map with addTiles(). Then we add the Pennsylvania counties with addPolygons() where we specify the areas boundaries color (color) and the stroke width (weight). We fill the areas with the colours given by the color palette function generated with colorNumeric(), and set fillopacity to a value less than 1 to be able to see the background map. We use colorNumeric() to create a color palette function that maps data values to colors according to a given palette. We create the function using the parameters palette with the color function that values will be mapped to, and domain with the possible values that can be mapped. Finally, we add the legend by specifying the color palette function (pal) and the values used to generate colors from the palette function (values). We set opacity to the same value as the opacity in the areas, and specify a title and a position for the legend.

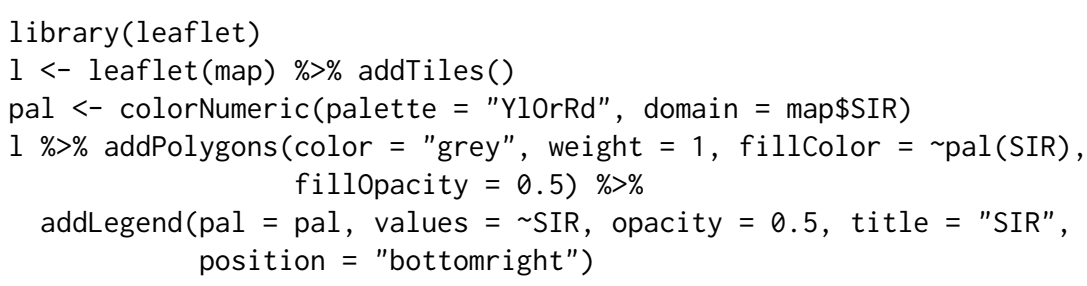

We can improve the map by highlighting the counties when the mouse hovers over them, and showing information about the observed and expected counts, SIRs, and smoking proportions. We do this by adding the arguments highlightoptions, label and labeloptions to addPolygons(). We choose to highlight the areas using a bigger stroke width (highlightoptions (weight $=4)$ ). We create the labels using HTML syntax. First, we create the text to be shown using the function sprintf() which returns a character vector containing a formatted combination of text and variable values and then applying htmltools: :HTML() which marks the text as HTML. In labeloptions we specify the labels style, textsize, and direction. Possible values for direction are left, right and auto and this specifies the direction the label displays in relation to the marker. We choose auto so the optimal direction will be chosen depending on the position of the marker.

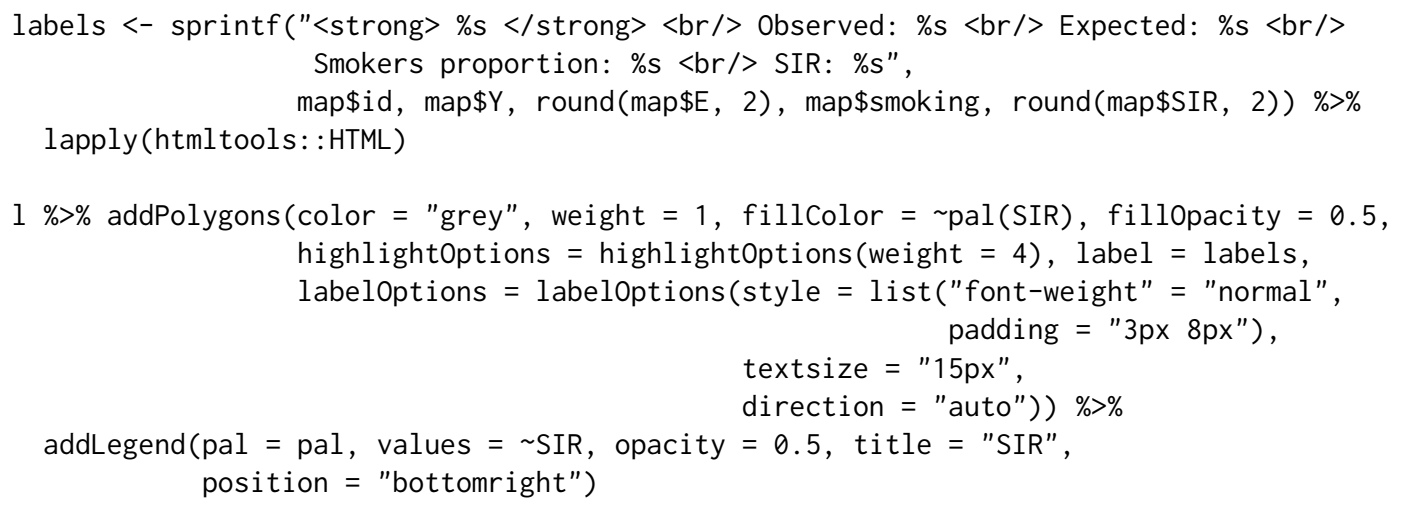

Figure 1 shows a snapshot of the interactive map created using leaflet showing the SIRs in the Pennsylvania counties. We can examine the map and see which counties have SIR equal to 1 indicating observed counts are the same as expected counts, and which counties have SIR greater (or smaller) than 1, indicating observed counts are greater (or smaller) than expected counts.

This map gives a sense of the disease risk across Pennsylvania. However, SIRs are misleading and insufficiently reliable in counties with small populations. In contrast, model-based approaches enable to incorporate covariates and borrow information from neighboring counties to improve local estimates, resulting in the smoothing of extreme values based on small sample sizes. In the next section we will show how to obtain disease risk estimates using a Bayesian model using INLA. 


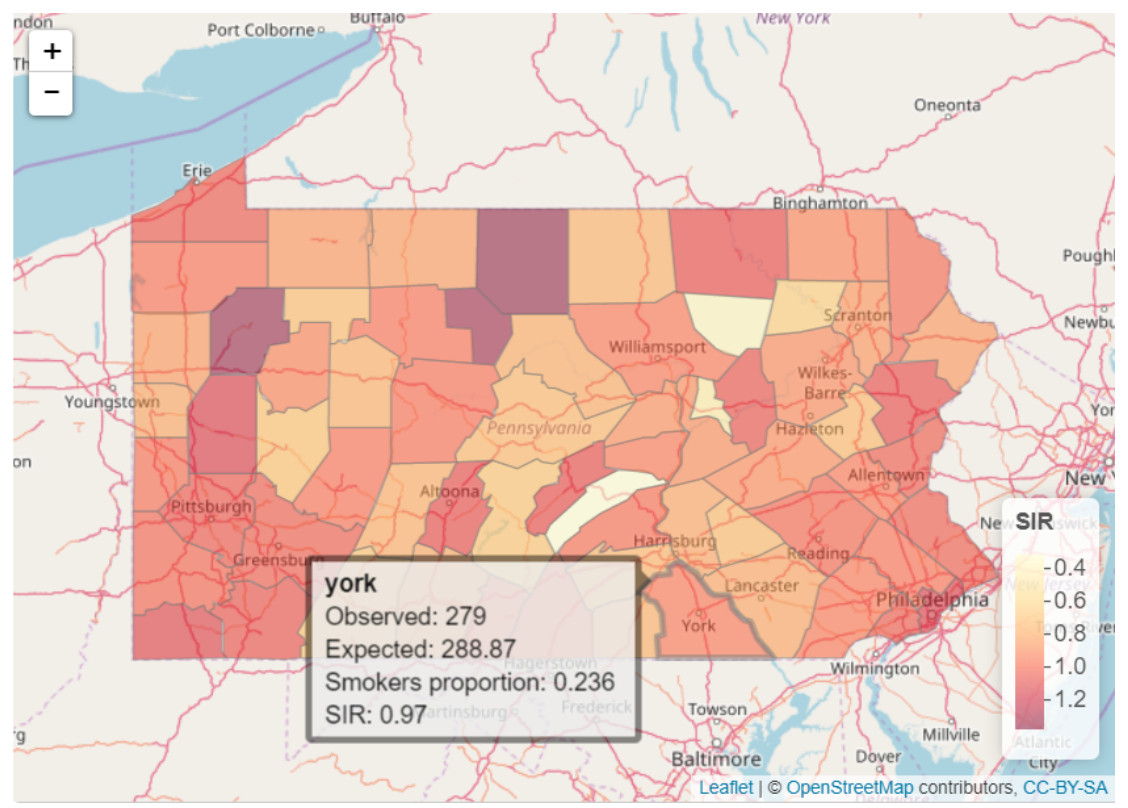

Figure 1: Snapshot of the interactive map created using leaflet showing the lung cancer SIRs in Pennsylvania counties in 2002.

\section{Modeling}

In this Section we specify the model for the data, and detail the required steps to fit the model and obtain the disease risk estimates using INLA.

\section{Model}

We specify a model assuming that the observed counts $Y_{i}$ are conditionally independently Poisson distributed,

$$
Y_{i} \mid \theta_{i} \sim \operatorname{Po}\left(E_{i} \times \theta_{i}\right), i=1, \ldots, n,
$$

where $E_{i}$ is the expected count and $\theta_{i}$ is the relative risk in area $i$. The logarithm of $\theta_{i}$ is expressed as follows:

$$
\log \left(\theta_{i}\right)=\beta_{0}+\beta_{1} \times \text { smoking }_{i}+u_{i}+v_{i},
$$

where $\beta_{0}$ is the intercept, $\beta_{1}$ is the coefficient of the smokers proportion covariate, $u_{i}$ is an structured spatial effect, $u_{i} \mid \mathbf{u}_{-\mathbf{i}} \sim N\left(\bar{u}_{\delta_{i}}, \frac{\sigma_{u}^{2}}{n_{\delta_{i}}}\right)$, and $v_{i}$ is an unstructured spatial effect, $v_{i} \sim N\left(0, \sigma_{v}^{2}\right)$.

\section{Neighbourhood matrix}

We create the neighbourhood matrix needed to define the spatial random effect using the poly2nb() and the nb2INLA() functions of the spdep package (Bivand, 2017). First, we use poly2nb() to create a neighbours list based on areas with contiguous boundaries. Then, we use nb2INLA() to convert this list into a file with the representation of the neighbourhood matrix as required by INLA that is saved in the working directory. Then we read the file using the inla. read.graph() function of INLA, and store it in the object $g$ which we will later use for specifying the spatial disease model with INLA.

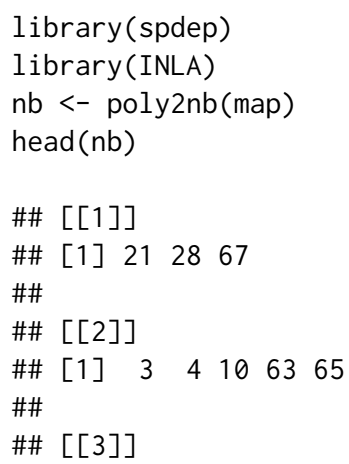




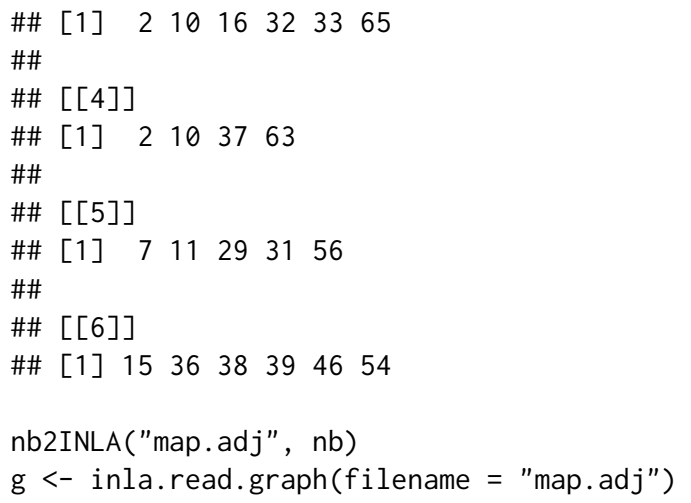

\section{Inference using INLA}

As stated in Section 2.4.3, the model includes two random effects, namely, $u_{i}$ for modeling spatial residual variation, and $v_{i}$ for modeling unstructured noise. We need to include two vectors in the data that denote the indices of these random effects. We call re_u the vector denoting $u_{i}$, and re_v the vector denoting $v_{i}$. We set both re_u and re_v equal to $1, \ldots, n$, where $n$ is the number of counties. In our example, $n=67$ and this can be obtained with the number of rows in the data (nrow(map@data)).

map\$re_u <- 1 : nrow(map@data)

map\$re_v <- 1 : nrow(map@data)

We specify the model formula by including the response in the left-hand side, and the fixed and random effects in the right-hand side. Random effects are set using $f()$ with parameters equal to the name of the variable and the chosen model. For $u_{i}$, we use model $=$ "besag" with neighbourhood matrix given by g. For $v_{i}$ we choose model $=$ "iid".

formula $<-Y \sim$ smoking $+f\left(r_{-} u\right.$, model = "besag", graph = g $)+f\left(r_{-} v\right.$, model $=$ "iid" $)$

We fit the model by calling the inla() function. We specify the formula, family, data, and the expected counts, and set control. predictor equal to list (compute = TRUE) to compute the posterior means of the linear predictors.

res <- inla(formula, family $=$ "poisson", data $=$ map@data, $\mathrm{E}=\mathrm{E}$,
control. predictor $=$ list $($ compute $=\mathrm{TRUE}))$

\section{Results}

We can inspect the results object res using summary().

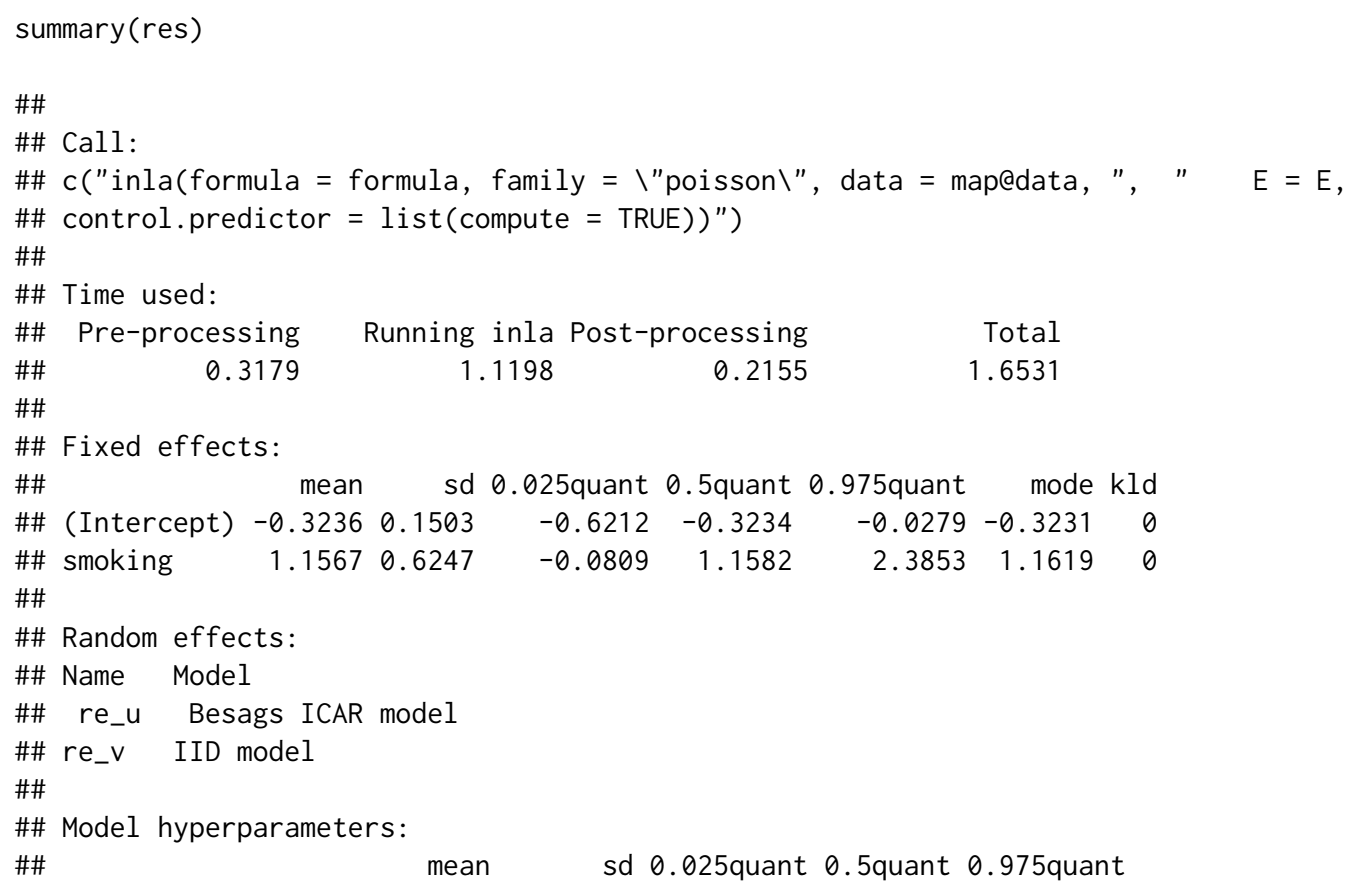




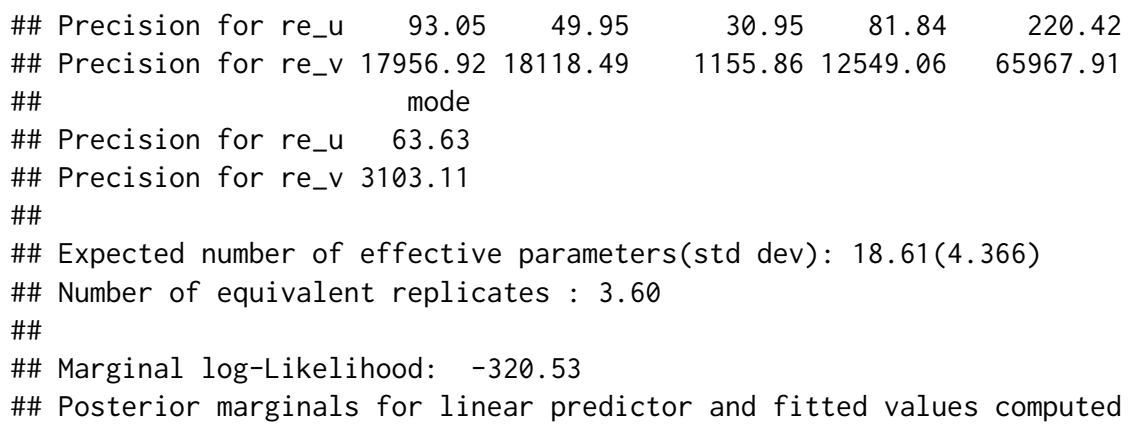

We see the intercept $\hat{\beta}_{0}=-0.3236$ with a $95 \%$ credible interval equal to $(-0.6212,-0.0279)$, and the coefficient of smoking is $\hat{\beta}_{1}=1.1567$ with a $95 \%$ credible interval equal to $(-0.0810,2.3853)$. This indicates that the smokers proportion has a positive although non significant effect on disease risk. We can plot the posterior distribution of the smoking coefficient. We do this by calculating a spline smoothing of the marginal distribution of the coefficient with inla.smarginal() and then plot it with ggplot() of ggplot2 package (Wickham and Chang, 2016) (see Figure 2).
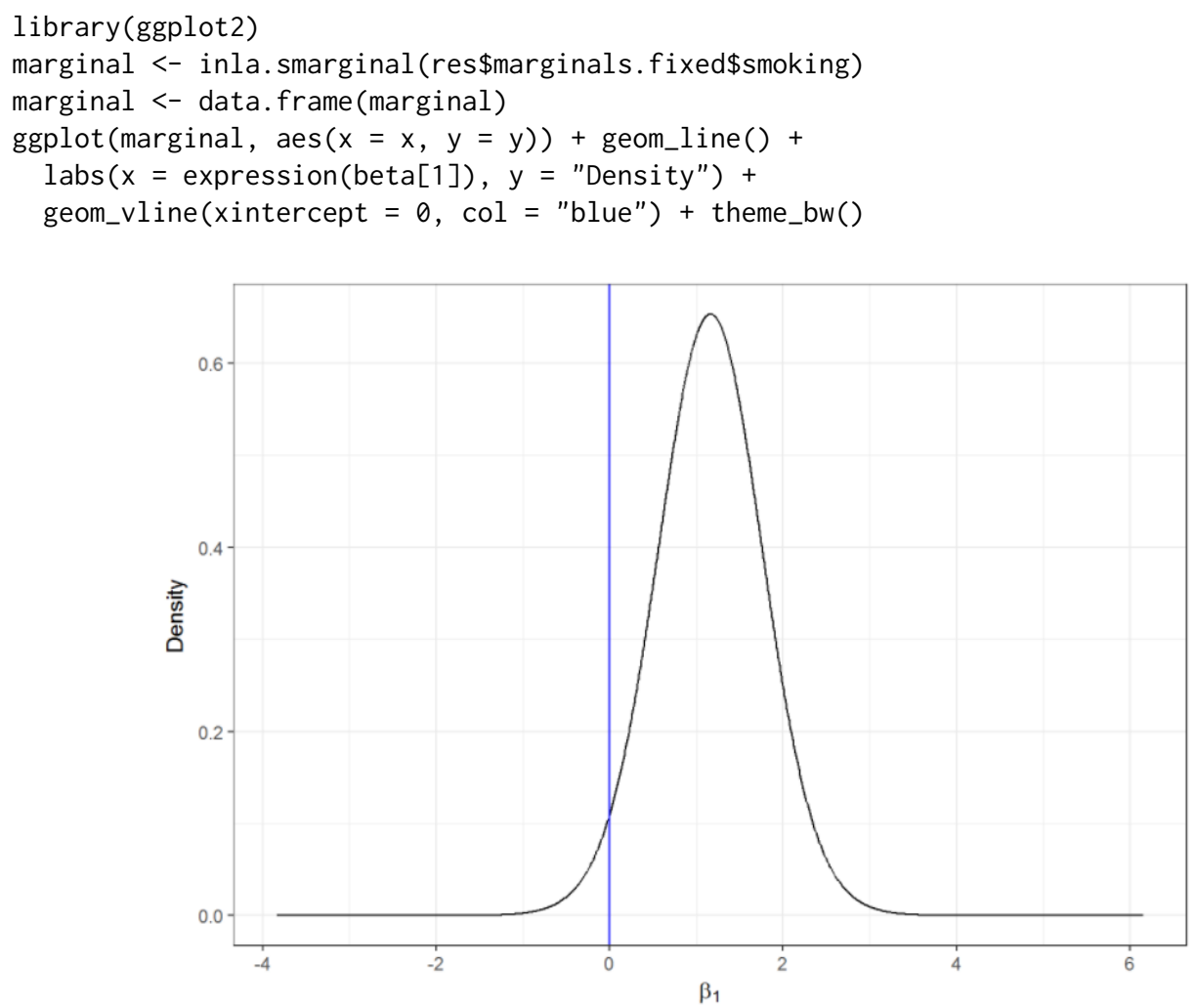

Figure 2: Posterior distribution of the coefficient of covariate smokers proportion.

The disease risk estimates and uncertainty for each of the counties are given by the mean posterior and the $95 \%$ credible intervals of $\theta_{i}, i=1, \ldots, n$ which are in the data frame res $\$$ summary. fitted. values. Here, column mean is the mean posterior and 0.025 quant and 0.975 quant are the 2.5 and 97.5 percentiles, respectively. We add these data to map to be able to make maps of these variables. We assign column mean to the estimate of the relative risk, and columns 0.025 quant and 0.975 quant to the lower and upper limits of $95 \%$ credible intervals of the risks.

head (res\$summary.fitted.values)

\begin{tabular}{lrrrrrr} 
& \multicolumn{2}{c}{ mean } & sd & 0.025 quant & 0.5 quant & 0.975 quant \\
\#\# fitted.Predictor.01 & 0.8793912 & 0.05856462 & 0.7633052 & 0.8797123 & 0.9943822 \\
\#\# fitted.Predictor.02 & 1.0597516 & 0.02768817 & 1.0067707 & 1.0592960 & 1.1153106 \\
\#\# fitted.Predictor.03 & 0.9632142 & 0.05186119 & 0.8555748 & 0.9649813 & 1.0612639 \\
\#\# fitted.Predictor.04 & 1.0270184 & 0.05121187 & 0.9270286 & 1.0267054 & 1.1289998 \\
\#\# fitted.Predictor.05 & 0.9076782 & 0.05497745 & 0.7978867 & 0.9081541 & 1.0156557
\end{tabular}




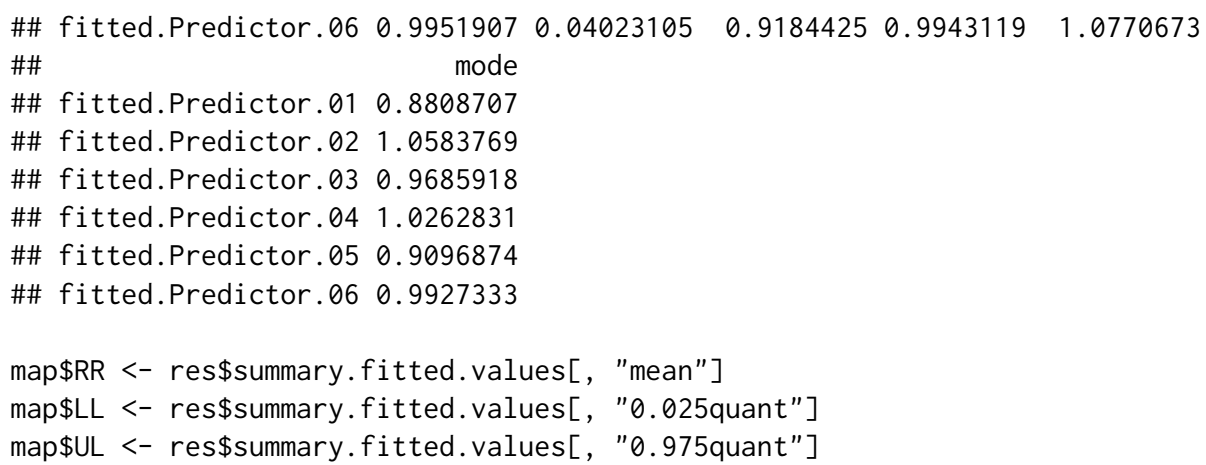

\section{Mapping disease risk}

We show the estimated disease risk in an interactive map using leaflet. In the map, we add labels that appear when mouse hovers over the counties showing information about observed and expected counts, SIRs, smokers proportions, RRs, and lower and upper limits of $95 \%$ credible intervals.

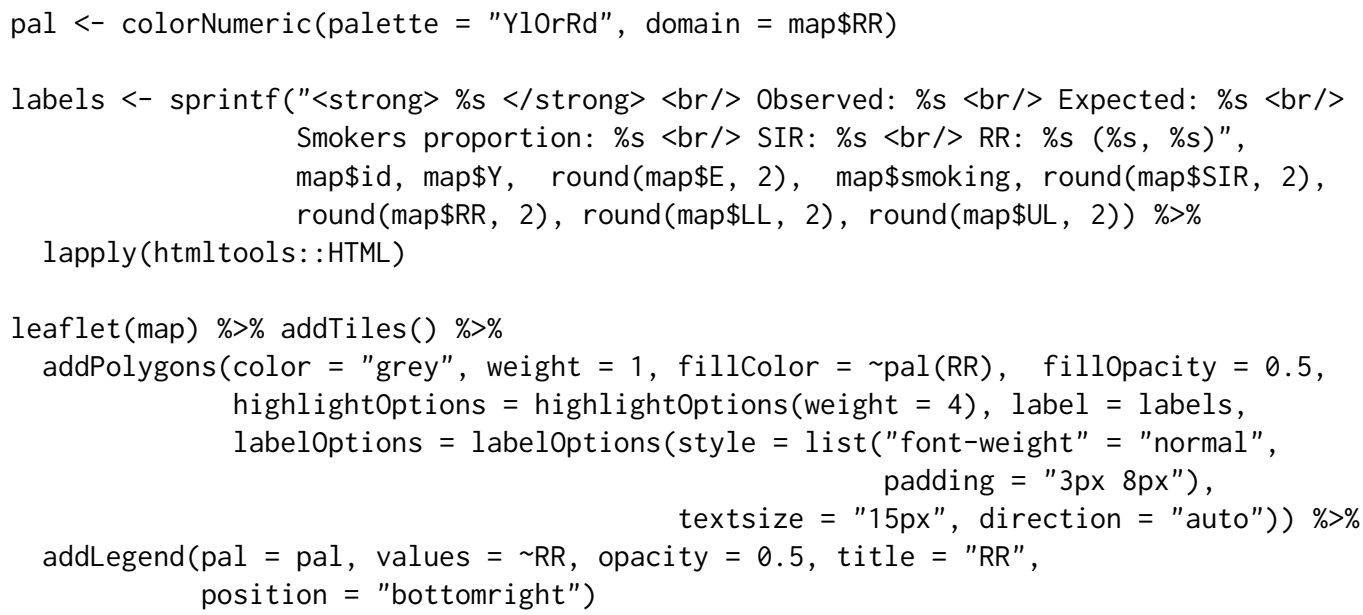

A snapshot of the interactive map created is shown in Figure 3. We observe counties with greater disease risk are located in the west and south east of Pennsylvania, and counties with lower risk are located in the center. The $95 \%$ credible intervals give a measure of the uncertainty in the risk estimates.

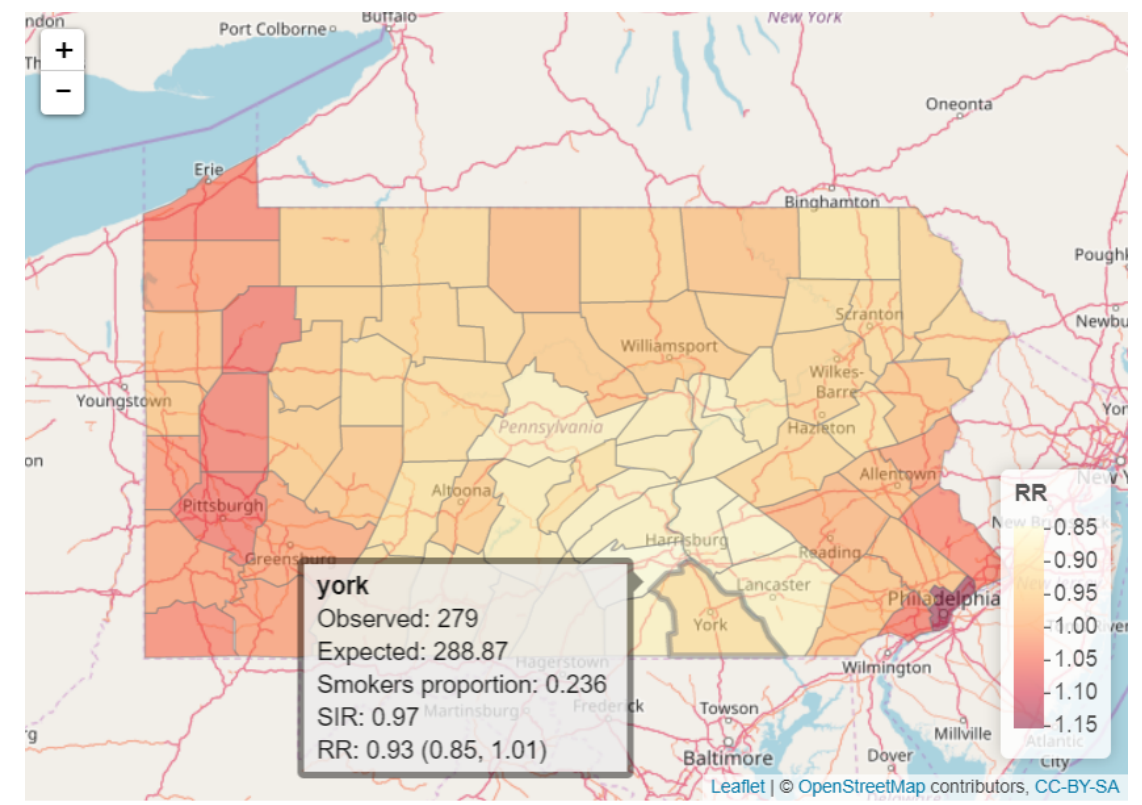

Figure 3: Snapshot of the interactive map created using leaflet showing the lung cancer RRs in Pennsylvania counties in 2002. 
By typing range (map@data $\$ S I R$ ) and range (map@data $\$ R R$ ) we see that the range of SIRs is much wider about 1 compared to the range of RRs: (0.32 to 1.37) versus (0.85 to 1.15). We can also see the shrinkage of the RRs toward 1 by comparing maps of SIRs and RRs created using the same scale on the SIR gradient legend (see Figure 4). For example, in the SIRs map, in the central, less populated part of the state, there are 3 counties with extreme high values (dark colour) and 3 counties with extreme low values (light colour). In the RRs map, these extreme values shrink toward values closer to 1.

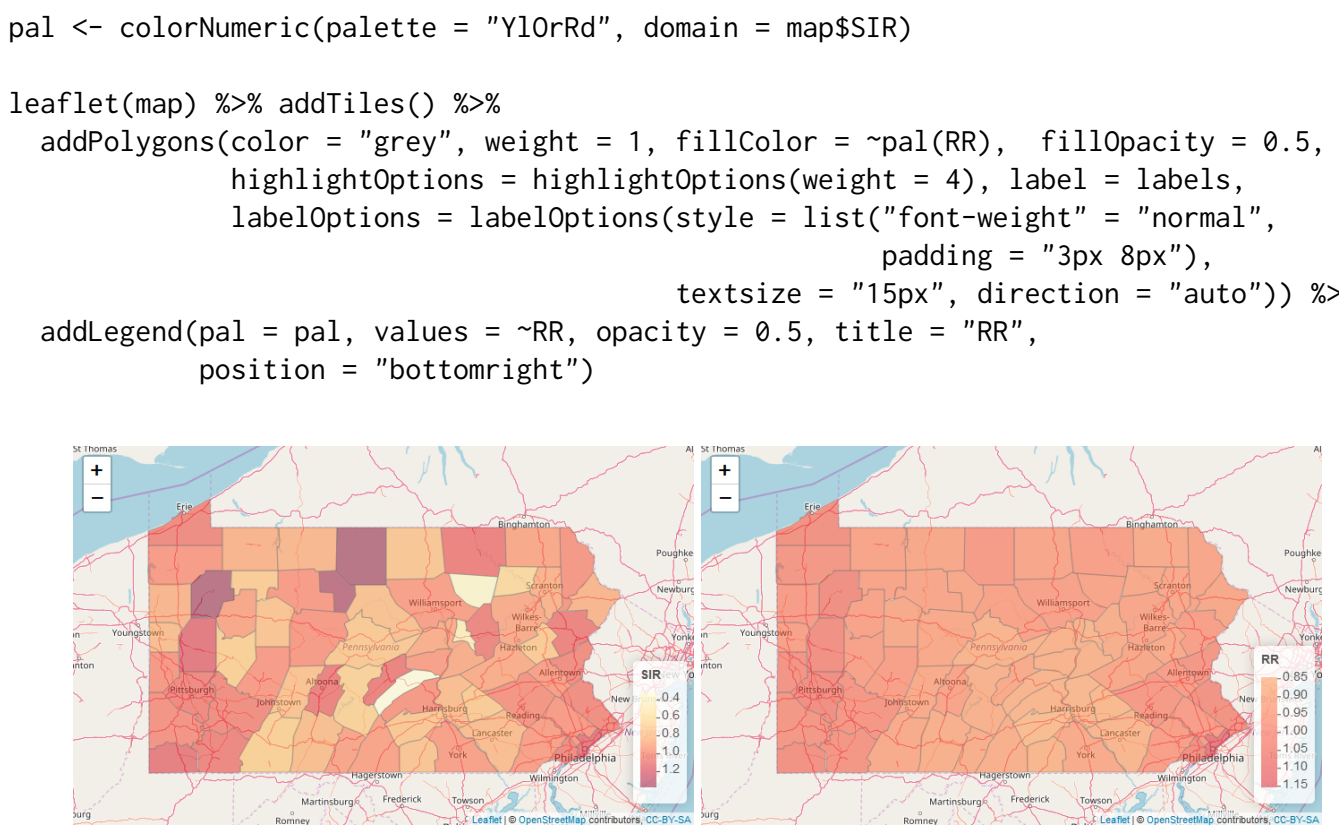

Figure 4: Snapshot of the interactive maps created using leaflet showing the lung cancer SIRs (left) and RRs (right) in Pennsylvania counties in 2002 using the same scale.

\section{Summary}

In this article we have shown how to obtain small area disease risk estimates, and generate interactive maps that help the understanding and interpretation of the results. First, we have introduced disease risk models using areal data, and have given an overview of the INLA package for performing Bayesian inference. Then, we have given a practical example where we have estimated lung cancer risk in Pennsylvania in 2002. We have conducted the analyses using several R packages such as spdep for spatial data manipulation, SpatialEpi for calculating the expected disease counts in the Pennsylvania counties, INLA for performing Bayesian inference, and leaflet and ggplot2 for visualization of the results.

One limitation of disease models based on areal data is that they are often subject to ecological bias. This bias occurs when associations obtained from analyses that use variables at an aggregated level lead to conclusions different from analyses that use the same variables measured at an individual level (Robinson, 1950). Therefore, whenever point data are available, it is preferable to use disease models without aggregating data and predict disease risk in a continuous surface (Moraga et al., 2017; Diggle et al., 2013).

It is also possible to use $\mathrm{R}$ to build tools to better communicate the results to stakeholders and the general public. For instance, summaries and maps of disease risk estimates can be presented in interactive dashboards using flexdashboard (Allaire, 2017), and web applications using shiny (Chang et al., 2017). One example of such web application is the SpatialEpiApp package (Moraga, $2017 a, b)$ which is useful for disease risk mapping and the detection of clusters. This is an easy to use application where users simply need to upload their data and click several buttons that execute the tasks and process the outputs, making spatial analysis methods accessible to multiple disciplines. SpatialEpiApp creates interactive visualizations by using the packages leaflet for rendering maps, dygraphs (Vanderkam et al., 2017) for plotting time series, and DT (Xie, 2016) for displaying data tables, and enables the generation of reports by using rmarkdown (Allaire et al., 2017). In conclusion, $\mathrm{R}$ represents an excellent tool for disease surveillance by enabling reproducible health data analysis. 


\section{Bibliography}

J. Allaire. Flexdashboard: R Markdown Format for Flexible Dashboards, 2017. URL https://CRAN.Rproject. org/package=flexdashboard. R package version 0.5. [p504]

J. Allaire, Y. Xie, J. McPherson, J. Luraschi, K. Ushey, A. Atkins, H. Wickham, J. Cheng, and W. Chang. Rmarkdown: Dynamic Documents for R, 2017. URL https://CRAN.R-project.org/ package=rmarkdown. R package version 1.8. [p504]

J. Besag, J. York, and A. Mollié. Bayesian image restoration with applications in spatial statistics (with discussion). Annals of the Institute of Statistical Mathematics, 43:1-59, 1991. URL https: //doi.org/10.1007/BF00116466. [p496]

R. Bivand. Spdep: Spatial Dependence: Weighting Schemes, Statistics and Models, 2017. URL https: //CRAN. R-project.org/package=spdep. R package version 0.6-13. [p500]

W. Chang, J. Cheng, J. Allaire, Y. Xie, and J. McPherson. Shiny: Web Application Framework for R, 2017. URL https://CRAN. R-project. org/package=shiny. R package version 1.0.5. [p504]

J. Cheng, B. Karambelkar, and Y. Xie. Leaflet: Create Interactive Web Maps with the JavaScript 'Leaflet' Library, 2017. URL http://rstudio.github.io/leaflet/. R package version 1.1.0.9000. [p495]

P. J. Diggle, P. Moraga, B. Rowlingson, and B. Taylor. Spatial and Spatio-Temporal Log-Gaussian Cox Processes: Extending the Geostatistical Paradigm. Statistical Science, 28(4):542-563, 2013. URL https://doi.org/10.1214/13-STS441. [p504]

A. E. Gelfand, P. J. Diggle, M. Fuentes, and P. Guttorp. Handbook of Spatial Statistics. Chapman and Hall/CRC, Boca Raton, Fl., 2000. [p496]

J. E. Hagan, P. Moraga, F. Costa, N. Capian, G. S. Ribeiro, E. A. Wunder Jr, R. D. Felzemburgh, R. B. Reis, N. Nery, F. S. Santana, D. Fraga, B. L. dos Santos, A. C. Santos, Q. A., W. Tassinari, M. S. Carvalho, M. G. Reis, P. J. Diggle, and A. I. Ko. Spatio-Temporal Determinants of Urban Leptospirosis Transmission: Four-Year Prospective Cohort Study of Slum Residents in Brazil. Public Library of Science: Neglected Tropical Diseases, 10(1):e0004275, 2016. URL https://doi .org/10.1371/ journal.pntd.0004275. [p495]

A. Y. Kim and J. Wakefield. SpatialEpi: Methods and Data for Spatial Epidemiology, 2016. URL https: //CRAN.R-project.org/package=SpatialEpi. R package version 1.2.2. [p497]

A. B. Lawson. Bayesian Disease Mapping: Hierarchical Modeling In Spatial Epidemiology. Chapman and Hall/CRC, Boca Raton, Fl., 2009. [p496]

B. G. Leroux, X. Lei, and N. Breslow. Estimation of disease rates in small areas: A new mixed model for spatial dependence. In M. E. Halloran and D. Berry, editors, Statistical Models in Epidemiology, the Environment, and Clinical Trials, The IMA Volumes in Mathematics and its Applications, pages 179-191. Springer-Verlag, New York, NY, 2000. [p495]

P. Moraga. SpatialEpiApp: A Shiny Web Application for the Analysis of Spatial and Spatio-Temporal Disease Data, 2017a. URL https://CRAN. R-project. org/package=SpatialEpiApp. R package version 0.3. [p504]

P. Moraga. SpatialEpiApp: A Shiny Web Application for the Analysis of Spatial and Spatio-Temporal Disease Data. Spatial and Spatio-temporal Epidemiology, 23:47-57, 2017b. URL https://doi .org/10. $1016 / j$.sste. 2017.08.001. [p504]

P. Moraga and M. Kulldorff. Detection of Spatial Variations in Temporal Trends with a Quadratic Function. Statistical Methods for Medical Research, 25(4):1422-1437, 2016. URL https://doi . org/10. 1177/0962280213485312. [p495]

P. Moraga and A. Ozonoff. Model-Based Imputation of Missing Data from the 122 Cities Mortality Reporting System (122 CMRS). Stochastic Environmental Research and Risk Assessment, 29(5):1499-1507, 2013. URL https://doi .org/10.1007/s00477-014-0974-4. [p495]

P. Moraga, J. Cano, R. F. Baggaley, J. O. Gyapong, S. Njenga, B. Nikolay, E. Davies, M. P. Rebollo, R. L. Pullan, M. J. Bockarie, D. Hollingsworth, M. Gambhir, and S. J. Brooker. Modelling the Distribution and Transmission Intensity of Lymphatic Filariasis in Sub-Saharan Africa Prior to Scaling up Interventions: Integrated Use of Geostatistical and Mathematical Modelling. Public Library of Science: Neglected Tropical Diseases, 8:560, 2015. URL https://doi .org/10.1186/s13071-015-1166-x. [p495] 
P. Moraga, S. Cramb, K. Mengersen, and M. Pagano. A Geostatistical Model for Combined Analysis of Point-Level and Area-Level Data Using INLA and SPDE. Spatial Statistics, 21:27-41, 2017. URL https://doi.org/10.1016/j.spasta.2017.04.006. [p504]

W. S. Robinson. Ecological Correlations and the Behavior of Individuals. American Sociological Review, 15(3):351-357, 1950. URL https://doi .org/10.2307/2087176. [p504]

H. Rue, S. Martino, and N. Chopin. Approximate Bayesian inference for latent Gaussian models using integrated nested Laplace approximations (with discussion). Journal of the Royal Statistical Society B, 71:319-392, 2009. URL https://doi.org/10.1111/j.1467-9868.2008.00700.x. [p495, 496]

H. Rue, S. Martino, F. Lindgren, D. Simpson, A. Riebler, E. T. Krainski, and G.-A. Fuglstad. INLA: Functions Which Allow to Perform Full Bayesian Analysis of Latent Gaussian Models Using Integrated Nested Laplace Approximations, 2017. R package version 17.06.20. [p495]

D. Vanderkam, J. Allaire, J. Owen, D. Gromer, P. Shevtsov, and B. Thieurmel. Dygraphs: Interface to 'Dygraphs' Interactive Time Series Charting Library, 2017. URL https://CRAN. R-project.org/ package=dyg raphs. R package version 1.1.1.4. [p504]

H. Wickham and W. Chang. Ggplot2: Create Elegant Data Visualisations Using the Grammar of Graphics, 2016. URL https: //CRAN. R-project. org/package=ggplot2. R package version 2.2.1. [p502]

Y. Xie. DT: A Wrapper of the JavaScript Library 'DataTables', 2016. URL https: //CRAN. R-project.org/ package=DT. R package version 0.2. [p504]

Paula Moraga

Centre for Health Informatics, Computing and Statistics (CHICAS)

Lancaster Medical School

Lancaster University

Lancaster, LA1 4YW

United Kingdom

ORCiD: 0000-0001-5266-0201

Webpage: https://paula-moraga.github.io/

p.e.moraga-serrano@lancaster.ac.uk 